\title{
Cloning and Expression of the Pathogenicity-Related Genes of Oidium heveae in the Infection Process
}

\author{
Yaqi Sun ${ }^{1}$, Peng Liang ${ }^{1}$, Qiguang $\mathrm{He}^{1}$, Wenbo Liu', Rong $\mathrm{Di}^{1{ }^{1,2}}$, Weiguo Miao ${ }^{1 *}$ and Fucong Zheng ${ }^{1 *}$ \\ ${ }^{1}$ Hainan Key Laboratory for Sustainable Utilization of Tropical Bioresource/College of Environment and Plant Protection, Hainan University, Haikou 570228, China \\ ${ }^{2}$ Department of Plant Biology, Rutgers, the State University of New Jersey, New Brunswick, New Jersey 08901, USA
}

\begin{abstract}
Oidium heveae B.A. Steinmann is a biotrophic fungus that infects rubber tree and causes powdery mildew disease, resulting in significant annual rubber yield losses worldwide. Researches on $O$. heveae in China had been limited on the cytological observation for the interaction of $O$. heveae with rubber tree, and the environment factors, such as temperature on the disease development. There had been scarce research on the infection mechanism of this important fungal pathogen at the molecular level. Pathogenicity-related genes of $O$. heveae are important for us to understand its infection process, which can potentially become targets for disease control. We have characterized eleven pathogenicity-related genes of $O$. heveae by genomics and transcriptomic studies. Four genes are involved in fungal metabolism, and the other three are related to fungal growth. Four genes were found to encode hypothetical proteins. The expression of these genes was further studied by qRT-PCR. Our results indicated that the expression of the metabolism-related Oh-PC2 gene encoding phosphoenolpyruvate carboxykinase (PEPCK) was up-regulated at 3 dpi (day post infection). Our data also demonstrated that at 3 dpi during the hyphae formation stage, the expression of all three fungal growth-related genes Oh-AAA-peroxin, Oh-RNP and Oh-Imp was up-regulated. Additionally, the expression of at least three $O$. heveae genes encoding hypothetical proteins was shown to increase at $3 \mathrm{dpi}$. Our results have provided novel insight to understanding the molecular mechanisms of $O$. heveae pathogenicity during its infection of rubber tree.
\end{abstract}

Keywords: Oidium heveae; Rubber tree powdery mildew; Pathogenicity-related genes; qRT-PCR

\section{Introduction}

Oidium heveae B. A. Steinmann infects rubber tree and causes powdery mildew, a worldwide disease in the rubber tree belt. O. heveae infection results in serious damage on the growth of rubber trees and significant economic loss in the rubber production. Cool and humid weather is favorable for powdery mildew disease outbreak on rubber trees. O. heveae is an obligate parasitic (biotrophic) fungus occurring on the leaf surface of rubber trees. The identification and classification of this fungus are still uncertain because there is no authoritative report of its morphology and no record of its teleomorphic stage $[1,2]$.

The powdery mildew fungus $O$. heveae of rubber tree damages rubber tree leaves, buds, shoots and inflorescence. It causes repeated infection on leaves throughout the year with conidial spores. Presently, the main disease control measures rely on new pesticides and resistant varieties [3]. In China, the studies of $O$. heveae have been limited to the cytology observation on the interaction of $O$. heveae with rubber tree, including the infection and development process and environmental factors such as temperature $[4,5]$. O. heveae is an obligate parasite, it cannot be cultured on medium [6]. There is very little known about the genes involved in its infection process [7].

Pathogenicity-related genes are important during pathogen infection process. They are involved in establishing the parasitic relationship with plant and undermining the normal physiological function of plant genes. They regulate a series of pathological processes, such as plant tropism, adsorption, invasion, colonization, expansion and destruction of the host [8]. At the early infection stage, conidiospores of $O$. heveae land on the leaf surface, similar to all powdery mildew fungi. The conidia started to form the germ tube and then form the appressoria. After forming an appressoria it began to penetrate the host and the haustoria develop within the cell, signaling a successful infection. After successful infection, the secondary hyphae continue branching out and spreading on the host surface. At last, the conidiophores are formed at the top of the stalk, finishing a full infection cycle [9]. This process is consisted of the initial, latent and post-infection periods.

Digital gene expression (DGE) tag profiling has been used to study the pathogenicity-related genes of Magnaporthe oryzae, the rice blast causal agent, which is the primary model system for elucidating various aspects of host plant-fungal pathogen interaction [10-12]. DGE method uses data of relative tag frequencies to show gene expression differences [13]. Transcriptomic analysis has also been used to study pathogenicity-related genes [5]. Yeonyee $\mathrm{Oh}$ et al. [5] reported the first comprehensive genome-wide transcript profile and the functional analysis of infection structure formation of Magnaporthe oryzae. Gene knockout and complementary strategies have also been used to study the function of pathogenicity-related genes [14]. SMART (Switching Mechanism At 5' end of the RNA Transcript) technology was used to construct normalized cDNA library from wheat powdery mildew (Blumeria graminis f. sp. Tritici) spores to study its gene function [15]

We have analyzed our transcriptome and genome data (unpublished) of $O$. heveae to search for the pathogenicity-related genes. We have identified 25 pathogenicity-related genes, from which 11 genes were selected based on the functions of their homologous proteins in other fungi. These 11 genes were cloned and were found to

*Corresponding authors: Weiguo Miao and Fucong Zheng, College of Environment and Plant Protection, Hainan University, Haikou, Hainan, China, Tel: +86-0898662-70229; Fax: +86-0898-661-92915; E-mail: weiguomiao1105@126.com, zhengfucong@126.com

Received March 18, 2015; Accepted June 15, 2015; Published June 25, 2015

Citation: Sun Y, Liang P, He Q, Liu W, Di R, et al. (2015) Cloning and Expression of the Pathogenicity-Related Genes of Oidium heveae in the Infection Process. J Plant Pathol Microbiol S1: 001. doi:10.4172/2157-7471.S1-001

Copyright: $\odot 2015$ Sun Y, et al. This is an open-access article distributed under the terms of the Creative Commons Attribution License, which permits unrestricted use, distribution, and reproduction in any medium, provided the original author and source are credited. 
belong to three categories, the metabolism- and growth-related genes, and genes encoding hypothetical proteins [5]. The expression of these genes during different infection stages was further analyzed by qRTPCR and compared to the transcriptomic data. This study has provided unprecedented insights to the pathogenicity-related genes of $O$. heveae during infection and disease development stages. This research is a basical work for the further function study of $O$. heveae.

\section{Materials and Methods}

\section{Strains of Oidium heveae}

Oidium heveae B.A.Steinmann strain HO-73 was collected from the rubber tree forest in Danzhou, Hainan. It was isolated by the "single spot" [16] method from rubber tree and deposited in the Molecular Plants Pathological Laboratory of the Environment and Plant Protection College in Hainan University. O. heveae HO-73 was inoculated onto cultivar Reyan 7-33-97 for the continuous passage culture (Table 1).

\section{RNA sample preparation and cDNA synthesis}

Total RNA was extracted from Oidium heveae at four infection stages, $0,1,3$ and $30 \mathrm{dpi}$ (day post infection), using Trizol (Life Technologies, Carlsbad, CA, USA) (Wan et al., [11]). The corresponding cDNA was generated by reverse transcription with the RevertAid First Stand cDNA Synthesis Kit (Thermo Fisher Scientific Inc., USA).

\section{Gene ontology and functional annotation}

Due to the lack of information on the functions of $O$. heveae genes, we used GO (Gene Ontology, http://geneontology.org/) to predict the functions of $O$. heveae genes related to pathogenicity. GO has been successfully used to provide structured, functional annotation of the rice blast pathogen Magnaporthe oryzae [11].

\section{Cloning the $O$. heveae pathogenicity-related genes}

Degenerate primers were designed with the software Primer Premier 5.0. The primers in Table 2 were used to amplify 11 of the pathogenicity-related genes from $O$. heveae DNA. Reaction conditions were as follows: $94^{\circ} \mathrm{C} 4 \mathrm{~min} ; 94^{\circ} \mathrm{C} 1 \mathrm{~min}, 50^{\circ} \mathrm{C} 30 \mathrm{sec}, 72^{\circ} \mathrm{C} 30 \mathrm{sec}$, for 30 cycles; $72^{\circ} \mathrm{C} 7 \mathrm{~min}$. The reaction system was as follows: Forword/ Reverse Primer $1 \mu \mathrm{l}$; DNA $2 \mu \mathrm{l}$; ddH $\mathrm{d}_{2} \mathrm{O} 6.5 \mu \mathrm{l}$; Mix $9.5 \mu \mathrm{l}$.

\section{The expression profile of $O$. heveae pathogenicity-related genes}

RNA was extracted from $O$. heveae at four infection stages. Quantitative RT-PCR (qRT-PCR) was used to quantify the expression levels of pathogenicity-related genes with ABI 7500 (Applied Biosystems, Foster City, CA, USA) and SYBR०RR Premix Taq ${ }^{\mathrm{TM}}$ kit
(Takara, Japan). The primers are shown in Table 3. 18s rRNA gene was selected as the internal reference. Reaction conditions were as follows: $95^{\circ} \mathrm{C} 30 \mathrm{sec} ; 95^{\circ} \mathrm{C} 5 \mathrm{sec}, 55^{\circ} \mathrm{C} 30 \mathrm{sec}, 72^{\circ} \mathrm{C} 30 \mathrm{sec}, 40$ cycles.

\section{Results}

\section{Identification of eleven pathogenicity-related genes}

The genomic sequences of $O$. heveae (our unpublished data) were uploaded to the online software DAVID to confirm the GO categories. Those sequences that received the GO number of 0009405 were identified as pathogenesis-related sequences. Our unpublished transcriptomic data demonstrated that some O. heveae genes were highly expressed at the peak ( $3 \mathrm{dpi}$ ) of the initial infection. Totally 11 genes were selected for further study. These genes were classified into three categories. The metabolism-related genes including Oh-EKA, Oh-G protein beta subunit/AC1, Oh-Gap and Oh-PC2 are involved in phosphorylation, kinase activity and amino acid metabolism during the host invasion. The growth-related genes including Oh-AAA peroxin, Oh-RNP and Oh-Imp are associated with the pathogen growth and development in the host, such as the formation of appressorium. Four other pathogenicity-related genes encode hypothetical proteins. The sequences of these 11 genes were used in the NCBI BLAST analysis to compare with other pathogenic fungi. The BLAST results confirmed that these $11 \mathrm{O}$. heveae sequences were homologous to the pathogenicity-related genes of other pathogenic fungi (Table 1).

\section{Cloning the $O$. heveae pathogenicity-related genes}

Based on the GO and BLAST analyses of these 11 genes, primers were designed (Table 2) and used to amplify parts of these 11 pathogenicity-related genes by PCR using the O. heveae DNA as the template. Partial cDNAs were obtained with lengths ranging from 250 to $1000 \mathrm{bp}$ (Figure 1). All cDNAs were sequence-confirmed to be identical to the genomic data of $O$. heveae.

Expression analysis of the O. heveae pathogenicity-related genes by qRT-PCR and comparison to the transcriptomic data

The expression of the 11 pathogenicity-related genes of $O$. heveae at different infection stages (0, 1, 3 and $30 \mathrm{dpi})$ was analyzed by qRT-PCR (Figure 2). For the four genes involved in the metabolism pathway, we found that the expression of $O h(O$. heveae)-EKA and $O h-G A P$ was not up-regulated at 1, 3 and $30 \mathrm{dpi}$ (Figure $2 \mathrm{~A}$ ). This qRT-PCR result was confirmed by our transcriptomic data (Figure $2 \mathrm{~B}$ ). The expression of Oh-G protein beta subunit/AC1 gene increased only slightly by 1.05 fold at 3 dpi (Figure 2A). The expression of Oh-PC2 gene, however, increased significantly by 15.79 -fold at 3 dpi (Figure 2A). Although the expression of $\mathrm{Oh}-\mathrm{PC} 2$ decreased slightly at $30 \mathrm{dpi}$, it was still as high as 13.53-fold (Figure 2A). The transcriptomic analysis seemed to

\begin{tabular}{|c|c|c|c|c|}
\hline Gene name & Description & Identity (\%) & Fungi & P-value \\
\hline Oh-EKA & EKA-like protein & 88 & Blumeria graminis & $5 e-32$ \\
\hline Oh-G protein beta subunit/AC1 & G-protein beta subunit & 97 & Blumeria graminis & 0.0 \\
\hline Oh-GAP & GTPase-activating protein & 64 & Blumeria graminis & $2 e-31$ \\
\hline Oh-PC2 & phosphoenolpyruvate carboxykinase & 60 & Magnaporthe grisea & $7 e-29$ \\
\hline Oh-AAA-peroxin & Reveals unique evolution of an obligate biotroph & 64 & Blumeria graminis & $5 e-117$ \\
\hline Oh-RNP & retrotransposon nucleocapsid protein & 44 & Metarhizium anisopliae & $5 e-103$ \\
\hline Oh-Imp & spore germination & 56 & Blumeria graminis & $5 e-91$ \\
\hline Hypothetical protein-1 & hypothetical protein & 47 & Pyrenophora teres & $4 e-13$ \\
\hline Hypothetical protein-2 & hypothetical protein & 39 & Penicillium digitatum & $5 e-12$ \\
\hline Hypothetical protein-3 & hypothetical protein & 66 & Penicillium digitatum & $9 e-05$ \\
\hline Hypothetical protein-4 & hypothetical protein & 76 & Blumeria graminis & $1 e-31$ \\
\hline
\end{tabular}

Table 1: The function and identity of amino acid of pathogenicity-related genes. The homologous genes of Oidium heveae by Blast. 
Citation: Sun Y, Liang P, He Q, Liu W, Di R, et al. (2015) Cloning and Expression of the Pathogenicity-Related Genes of Oidium heveae in the Infection Process. J Plant Pathol Microbiol S1:001. doi:10.4172/2157-7471.S1-001

Page 3 of 6

\begin{tabular}{|c|c|c|}
\hline Gene & Forward primer & Reverse primer \\
\hline Oh-GAP & TCGTTGTCCTCCTCGGTAG & AAGCGTGCTGAGAAATGG \\
\hline Oh-G protein beta & TGTCTTGATACTCGTCCTGTCGG & TATAGATGGAGCTCAGTTGCGGC \\
\hline Oh-PC2 & TGTGGAAACTTCACGGACTA & CATCAAGGCTTGCAGGAG \\
\hline Oh-RNP & CCACTCCACCCAGTCATC & CAGCGACAACTTGGCACCC \\
\hline Oh-EKA & AGGAAGTATGGGAGGTATGGACAA & CCGCCAACATCACTCCAACTTACAT \\
\hline Oh-AAA-peroxin & TGCCCGAACGCTATACCAACCAT & TATAGATGGAGCTCAGTTGCGGC \\
\hline Oh-Imp & TGTCTTGATACTCGTCCTGTCGG & TGGAACATAGACGAGCA \\
\hline Hypothetical1 protein-1 & TTGAAGACCTGGTGTAAGC & CTAAGTCTCCAGGGTAAT \\
\hline Hypothetical3 3 protein-3 & TGATGCCTAAACTCTTGT & TTGAAGCGTTTGGTCTGG \\
\hline Hypothetical4 protein-4 protein-1ppproproteinprotein & ATGGGTTCCTTGAGTCGG & TTGCCGGGAGAGAAGATGAGAAC \\
\hline
\end{tabular}

Table 2: Sequences of cloning primers used in this study. The cloning primers of the pathogenicity-related genes of Oidium heveae.

\begin{tabular}{|c|c|c|}
\hline Gene & Forward primer & Reverse primer \\
\hline $18 s$ & AAGCAAGCCTACGCTCTGG & GCTCCACCAACTAAGAACGG \\
\hline Oh-GAP & ССTTACACTCTTTTGССTTCC & GCCGTTGCATCATCCTTT \\
\hline Oh-G protein beta & TCGGGCTAACAGAAATGCG & GCTAAGGGAAAAGCGAGAAAG \\
\hline Oh-PC2 & TACTTTGCCCTCAGCCTTCG & TGCCTTGGGATCAGCGTC \\
\hline Oh-RNP & ACAGGGGCTGCCAAGAAG & CCAATCCTCCCGTAGAAGTAGA \\
\hline Oh-EKA & AGGAAGTATGGGAGGTATGGACAA & CAGCGTTCCCGGCTTTATTGTCT \\
\hline Oh-AAA-peroxin & CCAGCACTGTGGCTATCACT & CCCCAAGGCTCGACTAAC \\
\hline Hypothetical protein-1 & TTCCAACCAACCATCACC & GACCCCTATAATCCATCCAC \\
\hline Hypothetical protein-2 2protein-2 & CTGAGCTTAAATAACCACCCC & GTCCAAGTAACTACTATCCGTCCT \\
\hline Hypothetical protein-3 & CTTCTGCCCGTGAGTTATGC & GAGACTGCGAATCCCTGATC \\
\hline Hypothetical protein-4 & CAACGAAATCACGAAGAAGAATTG & TTGCCGGGAGAGAAGATGAGAACA \\
\hline
\end{tabular}

Table 3: Sequences of quantitative RT-PCR primer used in this study. The quantitative RT-PCR primers of the pathogenicity-related genes of Oidium heveae.

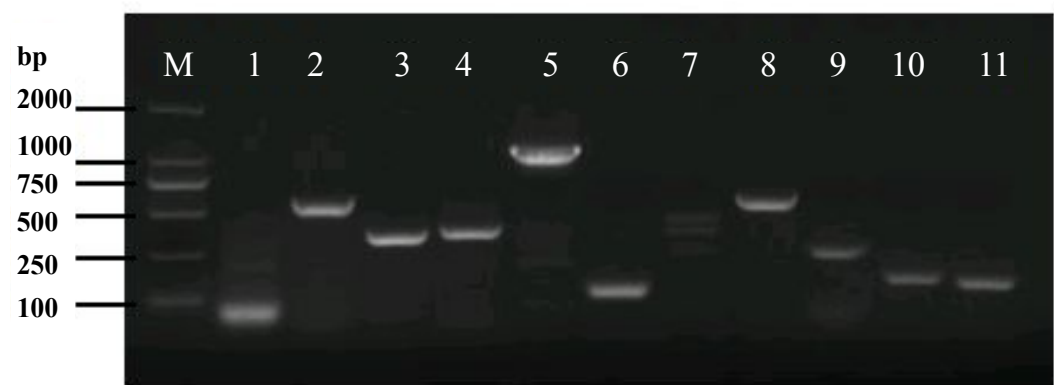

Figure 1: Cloning primer sequences used in this study. Cloning of $O$. heveae pathogenicity genes by PCR. 1. Oh-EKA;2. Oh-G protein beta subunit/AC1; 3. Oh-GAP; 4. Oh-PC2; 5. GOh-AAA-peroxin; 6. Oh-RNP; 7. Oh-Imp; 8. Hypothetical protein-1; 9. Hypothetical protein-2; 10. Hypothetical protein-3; 11 . Hypothetical protein-4.

show that $O h-P C 2$ 's expression increased at 3 dpi and decreased at 30 dpi (Figure 2B), but as a much lower level compared to the qRT-PCR result. This discrepancy could be due to the measurement error in the transcriptomic analysis, as it was performed only once. Out of these four genes, only $\mathrm{Oh}-\mathrm{PC} 2$ seemed to be highly up-regulated during $O$. heveae infection of rubber tree, indicating its significance in the metabolism of $O$. heveae.

For the O. heveae genes related to growth (Figure 2C), the expression of Oh-AAA-peroxin gene increased to 6.62 -fold at 3 dpi compared to $0 \mathrm{dpi}$ (Figure $2 \mathrm{C}$ ) and then decreased at $30 \mathrm{dpi}$. The $O h-R N P$ gene expression level increased to 7.65 -fold at $3 \mathrm{dpi}$ and then dropped to 3.43 -fold at $30 \mathrm{dpi}$ (Figure 2C), compared to its level at $0 \mathrm{dpi}$. The $\mathrm{Oh}$ Imp gene expression increased quickly to 4.0 -fold at $1 \mathrm{dpi}$, decreased to 2.83 -fold at $3 \mathrm{dpi}$ and continued to decline at $30 \mathrm{dpi}$. The expression trend of these three fungal growth-related genes as analyzed by qRTPCR was similar to our data in the transcriptomic analysis (Figure
2D). Day 3 is the mycelium growth period post infection for O. heveae, the up-regulation of Oh-AAA-eproxin, Oh-RNP and Oh-Imp gene expression underlines their importance for mycelium development.

Figure 2E shows that the gene expression of hypothetical protein-1, hypothetical protein-2 and hypothetical protein-3 increased to 4.32 , 3.79- and 27.69 -fold at 3 dpi. This result was confirmed by our transcriptomic data, however at different scales (Figure 2F). For the $\mathrm{Oh}$ hypothetical protein-4, although the GRT-PCR analysis did not show any gene expression changes at the infection stages, the transcriptomic data seemed to indicate a 7 -fold increase at $3 \mathrm{dpi}$ (Figure 2F).

\section{Discussion}

Prior to this study, little information is available on the gene functions of $O$. heveae. Genes responsible for the developmental stages of $O$. heveae had never been studied by the transcriptomic analysis. Transcriptome analysis at the early stage of infection has been done 
Citation: Sun Y, Liang P, He Q, Liu W, Di R, et al. (2015) Cloning and Expression of the Pathogenicity-Related Genes of Oidium heveae in the Infection Process. J Plant Pathol Microbiol S1:001. doi:10.4172/2157-7471.S1-001

A

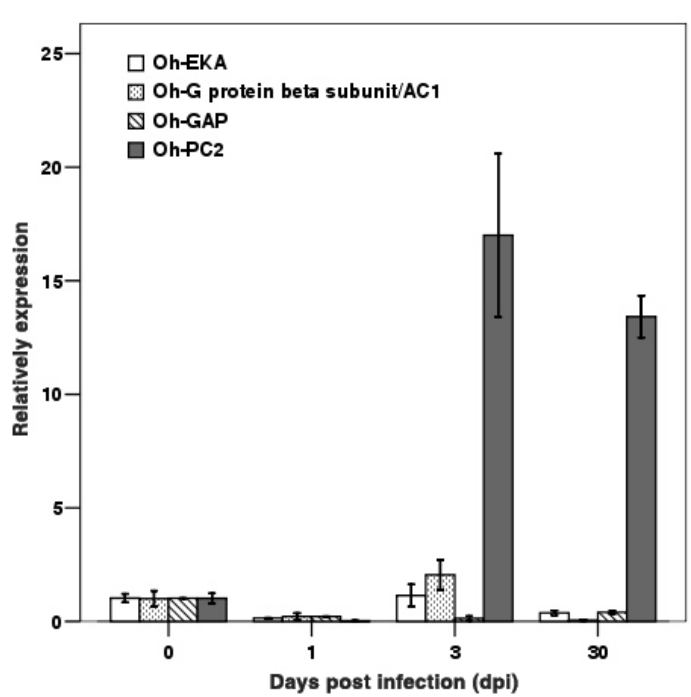

C

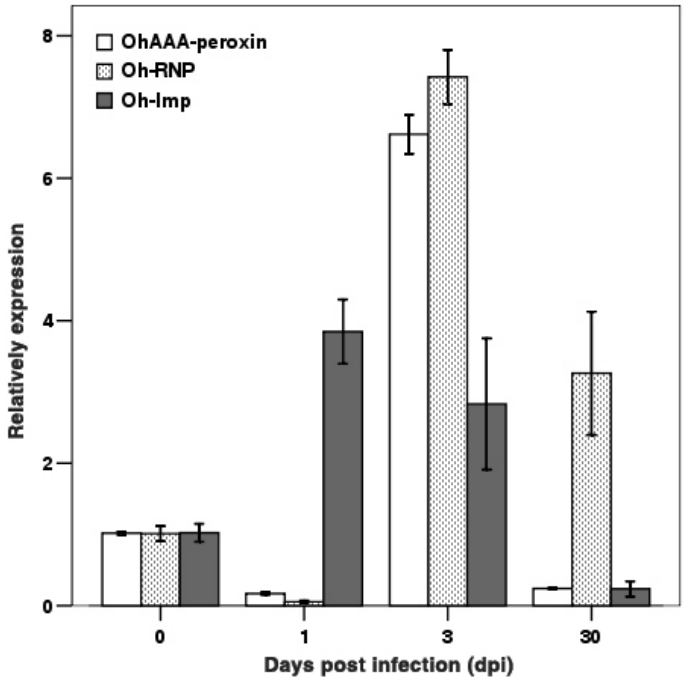

$\mathbf{E}$

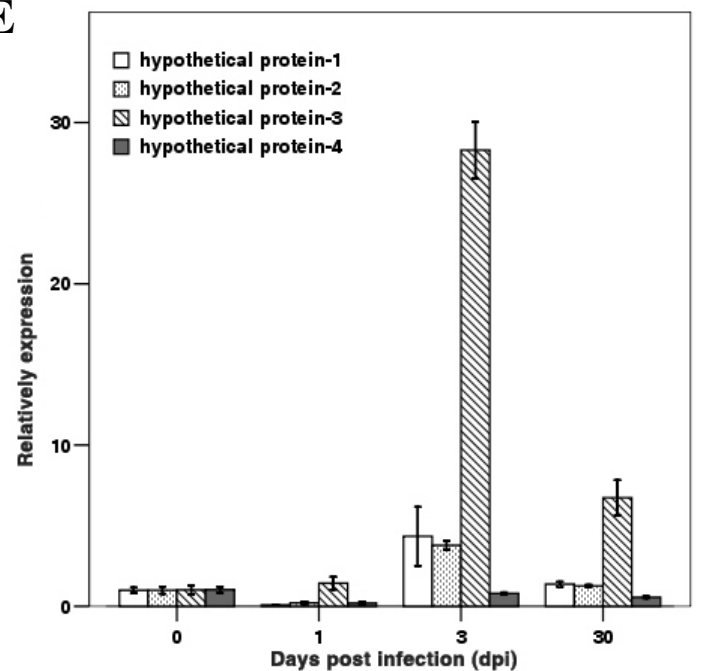

B

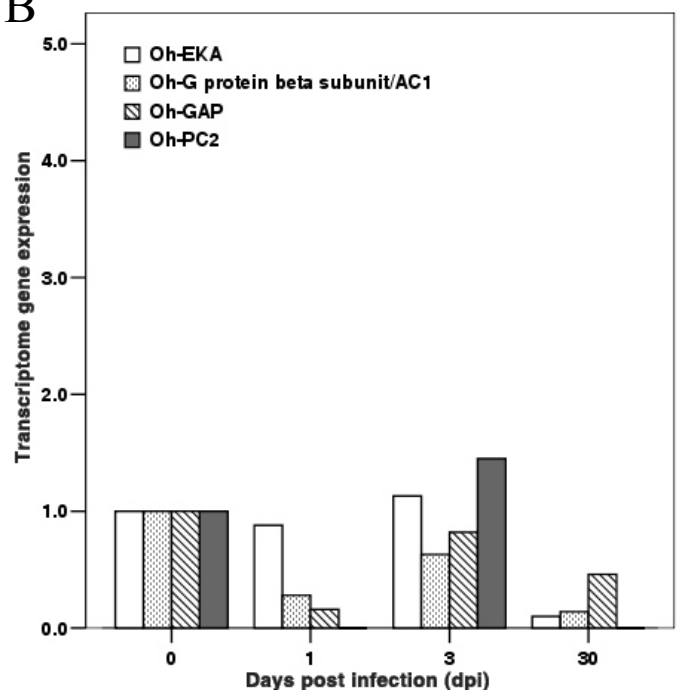

$\mathrm{D}$

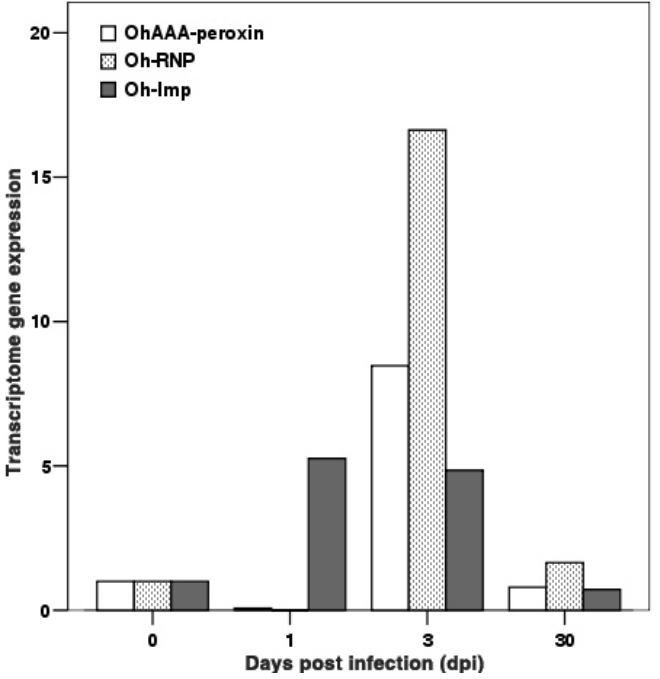

$\mathbf{F}$

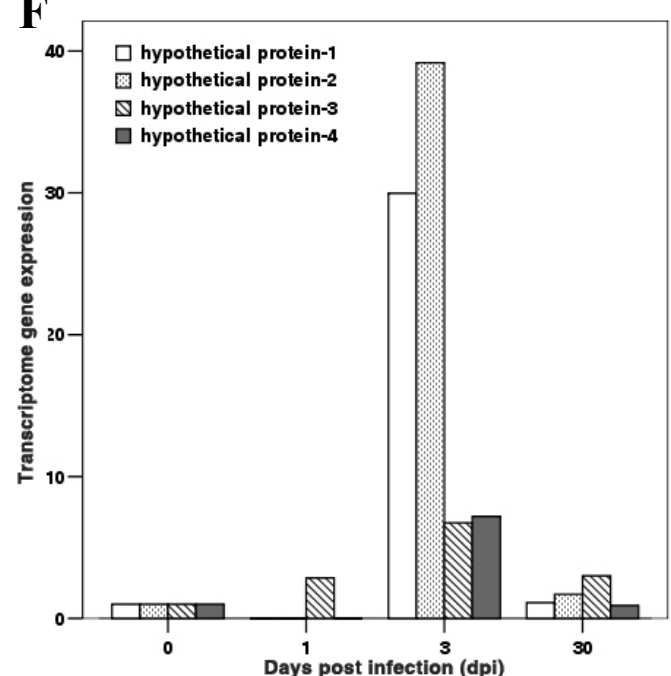

Figure 2: The relative expression of pathogenicity-related genes of $O$. heveae at different infection stages. (A) RT-qPCR analysis of metabolism-related genes; (B) transcriptomic data of metabolism-related genes; (C) RT-qPCR analysis of fungal growth-related genes; (D) transcriptomic data of fungal growth-related genes; (E) RT-qPCR analysis of hypothetical protein genes; $(F)$ transcriptomic data of hypothetical protein genes. 
Citation: Sun Y, Liang P, He Q, Liu W, Di R, et al. (2015) Cloning and Expression of the Pathogenicity-Related Genes of Oidium heveae in the Infection Process. J Plant Pathol Microbiol S1:001. doi:10.4172/2157-7471.S1-001

using ESTs and SAGE technology for some fungi, e.g. Magnaporthe oryzae [17]. In this work, we identified $11 \mathrm{O}$. heveae pathogenicityrelated genes through bioinformatic analysis of our genomic data (unpublished). We have also conducted transcriptomic analysis of $O$. heveae at $0,1,3$ and $30 \mathrm{dpi}$ (unpublished). When these 11 genes were analyzed for their expression levels at the same infection stages by qRT-PCR, we found our results were mostly similar to our $O$. heveae transcriptomic data (Figure 2).

We identified six out of eleven O. heveae genes that are homologous to those of Blumeria graminis, the barley powdery mildew causal agent (Table 1). They are Oh-EKA, Oh-G protein beta subunit/AC1, OhGAP, Oh-AAA-peroxin, Oh-Imp and the hypothetical protein-4. Both $M$. oryzae (the rice blast causal agent) and Blumeria graminis (the wheat powdery mildew causal agent) are obligatory parasitic fungi as O. heveae. Both B. graminis and O. heveae belong to Erysiphaceae. The expression patterns of $O$. heveae genes in the metabolism pathway and growth were similar to those in $M$. oryzae [11]. The fungal metabolism pathway-related genes especially those encoding protein kinase and phosphatase are important for growth, reproduction, information transmission and intrusion of the host [18]. The Oh-EKA is homologous with the EKA-like protein of $B$. graminis, with a role in genome expansion and gene loss in powdery mildew fungi $[18,19]$. The Oh-G protein beta subunit/AC1 is reported to have an effect on the appressorium formation in M. oryzae [20]. It is also important for the pathogenicity of other fungi, such as Cryphonectria parasitica, Puccinia recondita [21,22]. The Oh-GAP may function in the activation of the G-protein RACB. It is reported that G-protein RACB is required for full susceptibility of the leaf epidermis to the invasion by the biotrophic fungus $B$. graminis $f$. sp hordei. RACB is a common element of ingrowth of haustoria in leaf epidermal cells and the GTPase-activating protein is important for the process [23]. The fungal growth-related Oh-PC2 encodes phosphoenolpyruvate carboxykinase (PEPCK), which is an important enzyme required for gluconeogenesis [24]. Our data seemed to demonstrate that only the $\mathrm{Oh}-\mathrm{PC} 2$ gene expression was significantly up-regulated at $3 \mathrm{dpi}$ and $30 \mathrm{dpi}$, indicating its importance in the metabolism of $O$. heveae during infection of rubber tree.

The Oh-AAA-peroxin is homologous with a gene in the wheat powdery mildew genome. The $O h-R N P$ is homologous with the retrotransposon nucleocapsid protein of Metarhizium anisopliae (Table 1). It was reported that this gene is associated with pathogenhost interaction [25,26]. The Oh-Imp is homologous with the integral membrane protein of B. graminis, that is important for spore germination [19]. Our results indicated that the expression of these three genes were all up-regulated at $3 \mathrm{dpi}$, underlining their importance for $O$. heveae growth during the peak of the initial infection.

In the interaction of rubber tree with $O$. heveae, cell histology research indicates that hyphae grow 1 day after inoculation, mycelium starts to grow after 3 days, spores are produced after 30 days. Our data have demonstrated the different expression levels of different genes including those encoding hypothetic proteins at the initial and later stages of $O$. heveae infection on rubber tree.

While gene expression analysis is a useful tool to identify genes associated with a particular process, it is by no means definitive. Each gene needs to be validated for its function by knockout mutation analysis. In recent years, attention has been focused on pathogen effectors, many of which are secretory proteins or secondary metabolites. These effectors serve as virulence determinants or help shielding the pathogen from being detected by its host. In some cases, the plant host may recognize these effectors and activate its defense mechanisms. Our study has filled some gaps in the molecular study of
O. heveae and its pathogenicity, providing the groundwork for future studies on this important powdery mildew fungus.

\section{Acknowledgements}

This work was supported by grants from the National Key Basic Research Plan of China (2011CB111612), National Natural Science Foundation of China (31160359), National agricultural industrial technology system (CARS-34-GW8), Doctoral Fund of Ministry of Education (20104601110004), research and start-up capital of Hainan University (kyqd1006).

\section{References}

1. Limkaisang S, Komun S, Furtado EL, Liew KW Salleh B, et al. (2005) Molecular phylogenetic and morphological analyses of Oidium heveae, a powdery mildew of rubber tree. Mycoscience 46: 220-226.

2. Braun U, Cook RTA (2012) Taxonomic manual of the Erysiphales (Powdery Mildews). St Paul, MN, USA, APS Press.

3. Liu J (2010) Progress rubber tree powdery mildew. Tropical Agricultural Science \& Technology 33: 1-5.

4. Lu DJ (1982) Powdery mildew pathogen biology of rubber tree. Tropical 2: 6370

5. Oh Y, Donofrio N, Pan H, Coughlan S, Brown DE, et al. (2008) Transcriptome analysis reveals new insight into appressorium formation and function in the rice blast fungus Magnaporthe oryzae. Genome Biol 9: R85.

6. Tu M, Cai HB, Hua YW, Sun A, Huang H, et al. (2012) In vitro culture method of powdery mildew (Oidium heveae Steinmann) of Hevea brasiliensis. Afr $J$ Biotechnol 11: 13167-13172.

7. Gao HH, Liu XB, Luo DQ, Huang GX (2008) Extraction rubber powdery mildew (Oidium heveae Steinmann) genomic DNA. Tropical Agri Sci 27: 8-11.

8. Liu N, Hu JH, Jin BW (2013) Advances in genetic and non-toxic plant pathogen virulence genes. Acta Agriculturae Boreali-Sinica S1: 50-53.

9. Ridout CJ (2009) Powdery Mildews. Encyclopedia of life science 9: 1-8.

10. Anisimov SV (2008) Serial Analysis of Gene Expression (SAGE): 13 years of application in research. Curr Pharm Biotechnol 9: 338-350.

11. Li XS, Xu F, Wang HK, Lin FC (2013) Gene expression profiling related to hyphal growth in a temperature-sensitive mutant of Magnaporthe oryzae. J Integr Agr 12: 2189-2196.

12. Dean RA, Talbot NJ, Ebbole DJ, Farman ML, Mitchell TK, et al. (2005) The genome sequence of the rice blast fungus Magnaporthe grisea. Nature 434 980-986.

13. Hong LZ, Li J, Schmidt-Küntzel A, Warren WC, Barsh GS (2011) Digital gene expression for non-model organisms. Genome Res 21: 1905-1915.

14. Khan IA (2012) Identification, molecular cloning and characterization of pathogenicity-related genes in Magnaporthe oryzae genome. Hangzhou, China.

15. Li XL (2012) Wheat powdery mildew cDNA library construction, ESTs analysis of expression and Avra-10-like gene. Sichuan China, Sichuan Agricultura University.

16. Wang $\mathrm{CH}$, Wen $\mathrm{HK}$ (1997) A simple method for obtaining single-spore isolates of fungi. Bot Bull Acad Sin 38: 41-44.

17. Kim SG, Wang Y, Lee KH, Park ZY, Park J, et al. (2013) In-depth insight into in vivo apoplastic secretome of rice-Magnaporthe oryzae interaction. $\mathrm{J}$ Proteomics 78: 58-71.

18. Lee SC, Lee YH (1998) Calcium/calmodulin-dependent signaling for appressorium formation in the plant pathogenic fungus Magnaporthe grisea Mol Cells 8: 698-704.

19. Spanu PD, Abbott JC, Amselem J, Burgis TA Soanes DM, et al. (2010) Genome expansion and gene loss in powdery mildew fungi reveal tradeoffs in extreme parasitism. Science 330: 1543-1546.

20. Zhang HF (2011) Functional Analysis of the Magnaporthe oryzae G-protein and MAPK signaling pathway-related genes, Nanjing, China, Nanjing Agriculture University.

21. Kasahara S, Nuss DL (1997) Targeted disruption of a fungal G-protein beta subunit gene results in increased vegetative growth but reduced virulence. Mol Plant Microbe Interact 10: 984-993. 
Citation: Sun Y, Liang P, He Q, Liu W, Di R, et al. (2015) Cloning and Expression of the Pathogenicity-Related Genes of Oidium heveae in the Infection Process. J Plant Pathol Microbiol S1:001. doi:10.4172/2157-7471.S1-001

Page 6 of 6

22. Yang JJ, Li YN, Li X (2010) The expression of G-protein subunit in the Puccinia recondita interaction system and its relationship with disease resistance protein and active oxygen metabolism. Acta Agronomica Sinica 12: 2028-2034.

23. Hoefle C, Huesmann C, Schultheiss H, Börnke F, Hensel G, et al. (2011) A barley ROP GTPase activating protein associates with microtubules and regulates entry of the barley powdery mildew fungus into leaf epidermal cells. Plant Cell 23: 2422-2439.

24. Hynes MJ, Draht OW, Davis MA (2002) Regulation of the acuF gene, encoding phosphoenolpyruvate carboxykinase in the filamentous fungus Aspergillus nidulans. J Bacteriol 184: 183-190.

25. Hu X, Xiao G, Zheng P, Shang Y, Su Y, et al. (2014) Trajectory and genomic determinants of fungal-pathogen speciation and host adaptation. Proc Nat Acad Sci U S A 111: 16796-16801.

26. Beeley $F$ (1932) Effect of meteorological factors on the Occurrence of Oidium hevea is Malaya. J Rubb Res Inst Malay 2: 104-114. 